\title{
La donnée géographique aux frontières des organisations : approche socio-cognitive et systémique de son appropriation
}

\section{Matthieu Noucher}

\section{(2) OpenEdition \\ Journals}

Édition électronique

URL : http://journals.openedition.org/cdg/2203

DOI : $10.4000 /$ cdg. 2203

ISSN : 2107-7266

Éditeur

UMR 245 - CESSMA

Référence électronique

Matthieu Noucher, «La donnée géographique aux frontières des organisations : approche sociocognitive et systémique de son appropriation », Carnets de géographes [En ligne], 1 | 2010, mis en ligne le 01 octobre 2010, consulté le 23 septembre 2020. URL : http://journals.openedition.org/cdg/2203 ; DOI : https://doi.org/10.4000/cdg.2203

Ce document a été généré automatiquement le 23 septembre 2020.

\section{cc) $(1) \odot$}

La revue Carnets de géographes est mise à disposition selon les termes de la Licence Creative Commons Attribution - Pas d'Utilisation Commerciale - Pas de Modification 4.0 International. 


\title{
La donnée géographique aux frontières des organisations : approche socio-cognitive et systémique de son appropriation
}

\author{
Matthieu Noucher
}

1 Dans le contexte actuel de diffusion rapide des technologies de l'information géographique, les outils de la géomatique ne sont plus réservés à quelques techniciens mais touchent désormais l'ensemble des acteurs du territoire (urbanistes, géologues, forestiers, gestionnaires de réseaux, etc.). Face à cette multiplication de systèmes d'information géographique experts et devant les besoins croissants de visions intégrées du territoire, de nombreuses initiatives partenariales voient le jour. Leurs finalités tendent à évoluer progressivement: de la diffusion de référentiels géographiques à l'harmonisation voire la coproduction de données métier. On observe, ainsi, un basculement progressif des logiques de porter à connaissance vers des objectifs plus ambitieux de partage de connaissances.

2 Si les représentations spatiales, comme objets frontières, constituent des supports privilégiés des interactions entre acteurs du territoire, leur production et usages dans un contexte de réflexion collective participent surtout d'une réduction de la complexité des processus territoriaux à l'étude. La recherche proposée ici, vise à mettre en évidence que ces représentations sont utiles et pertinentes à analyser d'un point de vue cognitif, davantage sous l'angle de leur processus de production collectif, que comme les résultats finis de ces processus.

3 Ces travaux s'articulent autour de la formalisation des raisonnements sur l'espace par les technologies de l'information géographique, lesquelles sont envisagées comme des facilitateurs de la conception et de la communication des représentations spatiales. L'entrée proposée par l'usage des représentations spatiales dans des contextes inter- organisationnels (qui par nature sont également multi-acteurs et multi-échelles) consiste en particulier à étudier les solutions (outils, méthodes, 
compétences, organisations, approches) permettant de favoriser la conception, le partage, la communication et la traçabilité des représentations spatiales dans des démarches participatives de développement territorial.

4 La thèse visait plus spécifiquement à comprendre et analyser les enjeux de la coproduction de données géographiques. En effet, peut-on décider ensemble sans partager une compréhension commune des données qui servent au diagnostic? Comment le naturaliste s'approprie les données de l'urbaniste? Quels sont les facteurs qui influencent l'appropriation des données géographiques, fondements du diagnostic de l'existant? Les données géographiques nous permettent-elles de partager nos connaissances sur le territoire en jeu ou ne servent-elles, au contraire, qu'à exacerber nos différentes cultures-métier? Finalement, comment la donnée géographique peutelle être à la fois un objet de négociation collective (les réseaux se créent pour les échanger, les coproduire) tout en étant objet de représentation individuelle (i.e. traduction des modèles cognitifs territoriaux de leur producteur)? Aussi, l'objectif de la recherche était de comprendre le rôle joué véritablement par la donnée géographique dans la coordination et la coopération des acteurs du territoire.

5 Pour ce faire, nous avons choisi d'étudier les démarches d'appropriation des données géographiques. Elles nous permettent d'analyser comment, dans un contexte multiacteurs, une donnée peut trouver une place et un usage au sein d'une organisation qui ne l'a pas produite. Notre questionnement nous a amené à adopter une démarche qualitative, inductive et exploratoire où, selon les principes méthodologiques de la théorie ancrée, nos hypothèses de travail se construisent et se précisent au fil de la recherche. Quatre-vingt entretiens et une vingtaine d'observations nous ont, ainsi, permis d'effectuer des aller-retour entre observations empiriques et analyses théoriques afin d'en faire émerger progressivement différents éléments de compréhension.

6 L'approche socio-cognitive, consolidée au fil des observations, étudie le rôle de la donnée géographique en tant qu'objet-frontière de réseaux d'acteurs géographiquement, socialement et cognitivement distribués. Le cadre unificateur qu'offre la cognition socialement distribuée permet, en particulier, de comprendre le rôle d'artefact cognitif et collaboratif que peut jouer la donnée. Huit études de cas exploratoires (en France, en Suisse et sur des périmètres transfrontaliers) servent à identifier des trajectoires-types d'appropriation et les facteurs et processus socio-cognitifs associés.

7 Ces résultats sont prolongés par une approche systémique qui propose une vision intégrée et complémentaire des trajectoires-types d'appropriation. La mobilisation du méta-cadre systémique de Schwarz a mis en exergue l'enjeu essentiel des processus de médiation et a permis d'insister sur le caractère émergeant des connaissances partagées.

8 Enfin, un bilan de cette nouvelle façon d'aborder la géographie instrumentale envisage quelques leviers d'action, aussi bien au niveau des organisations (des logiques de compromis au consensus différencié), qu'au niveau des acteurs (de l'animateur au facilitateur) ou des outils (des ontologies pour standardiser aux ontologies pour négocier). Cette recherche offre, en effet, un regard différent sur la problématique du partage de données géographiques. La mobilisation d'une approche à la fois systémique et socio-cognitive permet d'envisager un nouveau rapport à la connaissance territoriale et à l'information géographique au sein des outils géomatiques. La thèse fournit ainsi 
des éléments de compréhension et d'intervention pour accompagner la transformation des dispositifs collaboratifs autour de l'information géographique en réseaux géomatiques apprenants, qu'il est également possible de qualifier de communautés de pratique. Ces communautés de pratique constituent, selon nous, l'un des fondements de l'Intelligence Territoriale.

\section{Fiche informative}

Thèse disponible aux Éditions Universitaires Européennes et sur : https:// www.epfl.ch/

\section{Discipline}

Géographie

\section{Directeurs}

François Golay (LaSIG - Ecole Polytechnique Fédérale de Lausanne)

Marie-Hélène de Sède-Marceau, co-directeur (ThéMA - Université de Besançon)

\section{Université}

École Polytechnique Fédérale de Lausanne

Membres du jury de thèse, soutenue le 23 mars 2009

François Golay (LaSIG - Ecole Polytechnique Fédérale de Lausanne)

Marie-Hélène de Sède-Marceau, co-directeur (ThéMA - Université de Besançon)

Jacques Lévy (CHOROS - Ecole Polytechnique Fédérale de Lausanne)

Thierry Joliveau (GDR MAGIS - CRENAM, Université de St Etienne)

Nicholas Chrisman (Géoïde - Université Laval, Québec)

\section{Situation professionnelle actuelle}

Consultant en Géomatique à Bordeaux au sein du cabinet IETI Consultants et chargé de cours dans les Universités d'Orléans, Bordeaux, Besançon et St Etienne.

\section{Contact}

matthieu.noucher[at]a3.epfl.ch

INDEX

Thèmes : Carnets de soutenances 